Panel Cambio Climático, Ambiente y Biosfera

\title{
Impacto Antropogénico sobre el Medioambiente
}

\author{
M.B. Espinosa \\ Consejo Nacional de Investigaciones Cientificas y Técnicas (CONICET). \\ E-mail:mespinosa@agro.uba.ar
}

La línea de tiempo de la historia evolutiva del planeta, muestra un lapso de 4.600 millones de años, el agua tiene 4.404 millones de años y los registros de seres vivos 4.280 millones de años. Sólo una fracción ínfima en esa línea de tiempo, le corresponde a la historia del Homo sapiens. La evolución de los homínidos (grupo "homininae") tiene unos 12 a 14 millones de años de historia, la familia Hominidae unos siete (7) millones de años de historia y hace sólo 2 ó 2,5 millones de años, aparecen en el registro fósil los primeros miembros del género Homo (Homo habilis). Los homínidos tardaron mucho tiempo en reconocer su capacidad para desarrollar actividades agropecuarias sedentarias. Hubo muchas generaciones de recolectores y cazadores nómadas antes de que se establecieran de modo sedentario. La transformación del ambiente natural, se incrementó drásticamente a partir de que se produjeron los primeros cultivos. Previamente, los homínidos, se proveían con lo que el ambiente natural ofrecía. La domesticación del fuego, de las plantas y de los animales, son hitos fundamentales. Los humanos modernos dominan el fuego desde hace unos 125.000 años. La domesticación de plantas y animales y la agricultura, tienen unos diez mil 0 doce mil años, ese es el tiempo en que los Homo sapiens modernos comenzaron a alterar sus dietas afectando profundamente sus medios de subsistencia a escala global. Hubo unos diez epicentros independientes donde se domesticaron plantas y animales (en Asia, América del Sur y África) permitiendo una mejor calidad de vida y un aumento de la población, pero causando modificaciones en el medioambiente y el ecosistema natural. Se han ocasionado impactos perjudiciales como la deforestación y desertificación, el aumento de la temperatura global, las modificaciones del régimen pluviométrico, la contaminación del aire y del agua, intervención en la geomorfología e hidrología así como cambios en la atmósfera y el clima. Finalmente también habrá que considerar la criosfera y su futuro.

Palabras Clave: evolución humana, fuego, agricultura, medioambiente.

\section{Pasado y Presente}

Podríamos graficar de forma simple, la línea de tiempo que refleja la historia evolutiva de la vida en el planeta, los primeros estadios corresponden a un lapso de 4.280 millones de años (se data el origen del planeta hace unos 4.600 millones de años y la presencia de agua tiene 4.404 millones de años). Sólo una fracción muy pequeña de esa línea de tiempo, refleja la proporción que le corresponde a la historia del Homo sapiens. Si tomáramos una línea de 30 centímetros, la historia evolutiva de nuestra especie, se reduciría a menos de 1 milímetro en el extremo final. La evolución de los homínidos se conoce con mucho detalle y el grupo "homininae" tiene unos 12 a 14 millones de años de historia. La familia Hominidae tiene unos siete (7) millones de años de historia sobre el planeta y hace tan solo 2 ó 2,5 millones de años, aparecen en el registro fósil los primeros miembros del género Homo (Homo habilis). Estos registros, muestran al género Homo junto con la diversificación de las coníferas y el ancestro del ganado (Bos primigenus) que 
evoluciona en la India. Durante la evolución de la población humana, se reconocen diversas fases del desarrollo cultural y se sabe que algunas especies de mamíferos como por ejemplo los cánidos han acompañado a los hombres "primitivos" y posiblemente hayan co-evolucionando en paralelo. Por dar un ejemplo, los Neandertales (Homo neanderthalensis) ya utilizaban el fuego y tenían herramientas con las que cazaban a sus presas hace entre 230. 000 y 28.000 años, al final del Pleistoceno. En este contexto, podemos pensar que ya los neandertales actuaban modificando el medio ambiente con herramientas. Podemos imaginar a los "primitivos" neandertales cazando mamuts (Mammuthus) y podríamos enfocar el problema como un problema de "co-evolución". Homo sapiens co-evoluciona con varias especies que se ven afectadas mutuamente en una interdependencia recíproca.

Los homínidos tardaron mucho tiempo en reconocer su capacidad para desarrollar actividades agropecuarias sedentarias, pasaron muchas generaciones de recolectores y cazadores nómadas hasta que se convirtieron en sedentarios. En ese sentido, el efecto transformador del ambiente natural, se incrementó drásticamente a partir del momento en que se comenzaron a producir los primeros cultivos. En etapas previas al sedentarismo, los primeros grupos de Homo sp. se proveían con lo que el ambiente natural ofrecía: frutas y hortalizas de estación y con suerte, de tanto en tanto algún huesito de mamut. Se ha determinado que los neandertales desaparecieron al competir por la comida con los Homo sapiens porque ambos basaban su dieta en mamuts y verduras crudas. Sin embargo si nuestra ascendencia reciente se encuentra fuera de África, podemos suponer sin peligro que llevamos ADN de Neanderthal, aproximadamente un 2\% (Human Evolution, Natural History Museum).

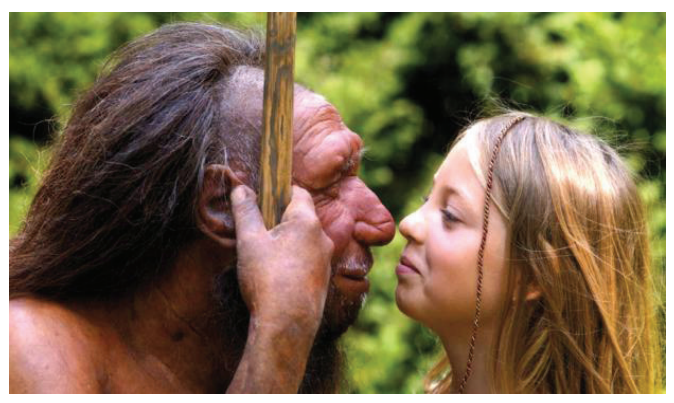

El control del fuego realizado por los primeros homínidos fue un punto de inflexión en la evolución cultural y biológica. El control del fuego permitió, entre otros avances una mejora en la nutrición favoreciendo el desarrollo cognitivo (se ha postulado que el cerebro pudo desarrollarse mejor gracias a la ingesta de alimentos cocidos que permitió un mejor aprovechamiento de las macromoléculas como proteínas, hidratos de carbono, etc.). La evidencia sobre el control del fuego por parte de humanos se encontró en Sudáfrica, la más antigua se remonta de 200.000 a 700.000 años. Los homínidos del paleolítico, tenían herramientas que les permitieron adaptarse al medio y al mismo tiempo pudieron ejercer una influencia sobre el ambiente. La domesticación del fuego, les permitió modificar los paisajes boscosos. Más allá del fuego natural, que se mostraba como una fuerza inexplicable (ciega, caprichosa, al azar) y pese a que el control del fuego insumió muchísimo trabajo, les proporcionó importantes beneficios. Poder prolongar la iluminación del día, mejorar la calefacción y la cocción de Ios alimentos les condujo a elevar el estándar de vida. En la actualidad, la mayor parte del fuego de nuestro planeta es de origen antropogénico y nosotros los humanos ya no somos el hombre primitivo porque la domesticación del fuego nos ha separado aún más de todas las especies relacionadas. Somos cada vez más parecidos a nosotros y más distantes de otras especies. Los distintos estadios del desarrollo humano han significado para el ambiente modificaciones que han tenido más 0 menos impacto. Se cree que en la actualidad, el impacto ejercido por las actividades humanas sobre el medioambiente, es de una magnitud sin precedentes.

\section{Deforestación y Desertificación}

La desertificación se define como "el proceso de transformación de tierra fértil en desierto, como resultado de la deforestación, la sequía o la agricultura inadecuada 0 inapropiada". En nuestro país, a fines del siglo XIX, Ernesto Tornquist (1842-1908) organizó la explotación de quebracho. El quebracho se exportaba a Inglaterra mediante el uso del ferrocarril. La deforestación del área de bosque seco de la región Chaqueña, devastó el ecosistema. Actualmente una de cada cinco partes del Planeta Tierra están amenazadas 
con la desertificación provocada por deforestación. Un caso de deforestación desafortunado es el de la "Amazonia" (se realizó para la Rodovía Transamazónica RB-230). Comenzada en la década de los años 70 dibujó un "tajo" en la selva amazónica que no ha conducido a los fines económicos ni agropecuarios pensados. "Siendo el lugar más biodiverso del planeta, la deforestación del Amazonas conduce a amenazas y extinciones de especies animales y vegetales. Las principales causas de la deforestación en la Amazonía son la conversión de tierras para la agricultura (ganado vacuno, también soja) prácticas en su mayoría ilegales" (Boekhout van Solinge, 2010). Las selvas representan un ecosistema muy vulnerable, mucho más difícil de recuperar que un pastizal.

\section{Aumento de la temperatura global}

El aumento de la temperatura global, debido al efecto invernadero provocado por los gases que se emiten durante la combustión, es el hito más relevante que cabe mencionar entre los efectos antropogénicos sobre el medioambiente. Las consecuencias del aumento de temperatura son de una enorme magnitud comprometiendo el descongelamiento de glaciares y capas de hielo polares. Como consecuencia de la fusión de capas de hielo en la Antártida, se producirá un incremento de unos $26 \mathrm{~mm}$ en el nivel del océano hacia el año 2100 (Schannwell et al., 2018). El incremento de la temperatura, a nivel global, debe ser tenido en cuenta junto a las modificaciones del medioambiente producidas como consecuencia de actividades y efectos locales. Los cultivos de maíz y soja transgénicos, en la región pampeana, contribuyen enormemente a la emisión de gases de efecto invernadero. En la región pampeana la emisión de gases con efecto invernadero, es mucho más alta que en las otras regiones del país (Arrieta et al., 2018).

\section{Modificaciones del régimen pluviométrico}

Los grandes cuerpos de agua artificiales como las represas, provocan una alteración importante del régimen pluvial (por ejemplo la represa hidroeléctrica de Itaipú). A su vez, el régimen pluvial tiene un efecto directo sobre la vegetación y la misma contribuye entre otras cosas a la oxigenación de la atmósfera. Se sabe que la mayor parte del oxígeno producido por fotosíntesis en el amazonas, se dirige al hemisferio norte gracias a las corrientes de aire, por ello un efecto nocivo para la selva amazónica, conducirá a un efecto pernicioso en otro sitio muy distante del planeta. En el caso de la región pampeana, se conoce el régimen pluviométrico y está bien caracterizado (Aliaga et al., 2016). El modo en que se modifique el ambiente tendrá un impacto directo en el régimen pluvial cuyas consecuencias pueden ser catastróficas inclusive para la agricultura.

\section{"Green Criminology"}

Se emplea el concepto de "Green Criminology" debido a la naturaleza interdisciplinaria de los problemas del medioambiente. Se hace referencia a la "Criminología Verde" cuando aparecen casos de lo que puede entenderse como ecocidio. Se ha propuesto que las leyes deben contemplar como crímenes las diversas formas de maltrato al medioambiente, el maltrato animal y todas las actividades que pudieran ocasionar pérdidas irremediables en la biodiversidad (White \& Graham, 2015). Hay algunas iniciativas importantes que pretenden contribuir al mantenimiento de la biodiversidad tal como la conocemos. Un proyecto conservacionista relevante es el "Millennium Seed Bank" del Jardín Botánico de Kew (https://www. kew.org/wakehurst/attractions/millennium-seed-bank). Tiene como objetivo, conservar el $25 \%$ de las especies de plantas del mundo para el año 2020. Al almacenar las semillas del mundo se proporciona un "seguro" contra la extinción. Algunos proyectos locales, de conservación de la biodiversidad se llevan a cabo en la Universidad Nacional del Sur bajo la tutela del Dr. Sergio Martín Zalba. Autores como Zalba, Estanga Mollica y otros, consideran que en las últimas décadas la actividad humana aceleró el movimiento de especies a tasas alarmantes empobreciendo la diversidad biológica del planeta y generando pérdidas cercanas al 5 por ciento del PBI global. El caldenal pampeano se ha empobrecido en biodiversidad por el uso de la ganadería intensiva y por el avance de la frontera agrícola. Es necesario conservar el bosque y fortalecer la aplicación de las leyes existentes. El análisis de las interacciones bióticas directas e indirectas durante la invasión de plantas exóticas, permite discernir los distintos factores que afectan la colonización 
(o invasión) de un sitio por especies alóctonas (Estanga Mollica, 2018). La adaptación juega un papel clave para algunas especies europeas que invaden zonas templadas (MacDougall et al., 2018).

\section{La rosa mosqueta, un ejemplo de "invasión"}

Un ejemplo de "colonización" por flora alóctona es el caso de la rosa mosqueta. La rosa mosqueta: Rosa eglanteria (sinónimo Rosa rubiginosa) pertenece a la familia de las rosáceas, originaria de Europa y común en el Reino Unido. Se utiliza tanto por su flor como por su fruto. Aquí se la puede hallar silvestre en la región sur de la Cordillera de los Andes y en el piedemonte mendocino. Su fruto, se utiliza para fabricar dulces y mermeladas y el aceite de sus semillas se utiliza en cosmética. Ha viajado traída por los colonos que se instalaron en las zonas del Lago Nahuel Huapi y ha competido con especies autóctonas colonizando la región.

\section{Efecto de la Contaminación del Aire sobre las Plantas y otros seres vivos}

Se conoce el efecto de algunos gases, producto de actividades como la combustión de carbón, sobre las plantas. Los niveles de los dos principales óxidos de nitrógeno, óxido nítrico (NO) y dióxido de nitrógeno $\left(\mathrm{NO}_{2}\right)$ son muy altos en la atmósfera del entorno urbano y en las proximidades del tráfico automovilístico y aeroportuario. Los óxidos de nitrógeno dan lugar a otros contaminantes fitotóxicos como el ozono y también son dañinos para la salud de las plantas. Producen lesiones no visibles que se reflejan en una disminución del crecimiento de la vegetación perdiendo productividad. Los efectos de las concentraciones atmosféricas de NOX como agentes fitotóxicos, no han sido revisadas con profundidad, pero se sabe que el crecimiento y la morfogénesis en plantas están mediados por hormonas y ocurren mediante procesos complejos en los que participan la longitud de onda de la luz y señalizaciones intracelulares. El óxido nítrico (NO) es un regulador del desarrollo de la planta que interactúa con la señalización hormonal. Puede en algunos casos tener un efecto inhibitorio del crecimiento de la raíz primaria como ocurre en el trigo (Lozano-Juste \& León, 2011). En la tabla, a continuación, se enumeran los principales gases cuyo efecto es nocivo para el follaje y la vegetación. Los gases y la materia particular (PM, particulate matter) que se encuentran en el aire como productos de diversas actividades humanas, están bien caracterizados y hay guías "on line" donde se establecen los límites sugeridos para los contaminantes del aire (Tabla I).

Tabla I. Contaminantes del aire cuyo efecto puede ser nocivo

\begin{tabular}{|c|c|c|c|}
\hline Compuesto & Molécula & $\begin{array}{l}\text { Máxima exposición } \\
\text { aceptable }\end{array}$ & Notas \\
\hline Dióxido de Azufre & $\mathrm{SO}_{2}$ & $\begin{array}{c}75 \mathrm{ppb} \text { (1 hora) } \\
0,5 \mathrm{ppm} \text { (3 horas) }\end{array}$ & $\begin{array}{c}\text { Aparece con las emisiones volcánicas, y es producido } \\
\text { químicamente. }\end{array}$ \\
\hline $\begin{array}{l}\text { Óxidos de } \\
\text { Nitrógeno } \\
\text { (productos de } \\
\text { combustión) }\end{array}$ & $\begin{array}{l}\mathrm{NO} \\
\mathrm{NO}_{2}\end{array}$ & $\begin{array}{c}30 \mathrm{mg} / \mathrm{m}^{3} \text { (8 horas) } \\
40 \mu \mathrm{g} / \mathrm{m}^{3} \text { (anual) } \\
200 \mu \mathrm{g} / \mathrm{m}^{3} \text { (por hora) }\end{array}$ & $\begin{array}{c}\text { Es una molécula de señalización pero a niveles de } 100 \\
\text { ppm, el óxido nítrico es inmediatamente peligroso para la } \\
\text { vida y la salud. }\end{array}$ \\
\hline Ozono & $0_{3}$ & $100 \mu \mathrm{g} / \mathrm{m}^{3}$ (por 8 horas) & $\begin{array}{l}\text { Ozonósfera necesaria ya que absorbe del } 97 \text { al } 99 \% \text { de } \\
\text { la radiación ultravioleta de alta frecuencia*. }\end{array}$ \\
\hline $\begin{array}{c}\text { Fluoruros } \\
\text { (Hexafluoruro de } \\
\text { azufre) }\end{array}$ & $\mathrm{SF}_{6}$ & $\begin{array}{l}\text { Es el gas con mayor } \\
\text { efecto "Green House" o } \\
\text { invernadero. }\end{array}$ & $\begin{array}{l}\text { Se usa como aislante en sistemas de producción de } \\
\text { electricidad. }\end{array}$ \\
\hline
\end{tabular}

*El ozono es estable sólo en determinadas condiciones de presión y temperatura. La ozonósfera es necesaria pero el contacto directo con ozono puede ser perjudicial para la salud. La materia particulada, también debe ser controlada. Se clasifica en PM 2.5 y PM 10. En ningún caso debe superar los $50 \mu \mathrm{g} / \mathrm{m}^{3}$ de exposición durante 24 horas. La materia particulada se produce como subproducto de las actividades de construcción. 
Desafortunadamente, han quedado varios aspectos importantes sin considerar. Mencionaremos que también deben ser tenidos en cuenta el impacto de la actividad humana sobre el agua, los efectos de la actividad humana sobre la geomorfología y la hidrología así como el impacto sobre la atmósfera y el clima. Finalmente también debe considerarse con especial atención la criósfera y su futuro.

\section{Referencias bibliográficas}

- Aliaga, V. S.; Ferrelli, F.; Alberdi-Algarañaz E. D.; Bohn, V. Y.; Piccolo, M. C. (2016) Distribution and variability of precipitation in the Pampas, Argentina. Cuadernos de Investigación Geográfica, [S.I.], v. 42, n. 1, p. 261-280, ISSN 1697-9540.

- Arrieta E.M.; Cuchietti, A.; Cabrol, D.; González, A.D. (2018) Greenhouse gas emissions and energy efficiencies for soybeans and maize cultivated in different agronomic zones: A case study of Argentina, Science of The Total Environment, Volume 625, Pages 199-208,

- Boekhout van Solinge (2010) Deforestation Crimes and Conflicts in the Amazon. T. Crit Crim 18: 263-277. https://doi.org/10.1007/s10612-010-9120-x

- Estanga Mollica, M. E. (2018) "Importancia de las interacciones bióticas directas e indirectas en la invasión de plantas exóticas" Tesis de Doctorado, Escuela para Graduados de la FA-UBA.

- Lozano-Juste, J., \& León, J. (2011). Nitric Oxide Regulates DELLA Content and PIF Expression to Promote Photomorphogenesis in Arabidopsis. Plant Physiology, 156(3), 1410-1423. http://doi.org/10.1104/ pp.111.177741

- MacDougall, A. S., McCune, J. L., Eriksson, 0. , Cousins, S. A., Pärtel, M. , Firn, J. and Hierro, J. L. (2018), The Neolithic Plant Invasion Hypothesis: the role of preadaptation and disturbance in grassland invasion. New Phytol. . doi:10.1111/nph.15285

- Schannwell, C., Cornford, S., Pollard, D., and Barrand, N. E.: Dynamic response of Antarctic Peninsula Ice
Sheet to potential collapse of Larsen C and George VI ice shelves, The Cryosphere, 12, 2307-2326, https:// doi.org/10.5194/tc-12-2307-2018, 2018.

- White, R., Graham, H. (2015) Greening Justice: Examining the Interfaces of Criminal, Social and Ecological Justice. Brit. J. Criminol. 55, 845-865 